\title{
Mejora de la funcionalidad física y emocional tras un programa de intervención multicomponente en mujeres con cáncer de mama
}

\section{Improvement of physical and emotional functionality after a multicomponent intervention program in women with breast cancer}

Fecha de recepción: 10-06-2019

Fecha de aceptación: 07-11-2019
Lorena Gutiérrez Hermoso

Universidad Rey Juan Carlos

Lilian Velasco Furlong Universidad Rey Juan Carlos Elisabeth Berzal Pérez Universidad Rey Juan Carlos Lorena Salas Costumero Universidad Rey Juan Carlos

Sofia Sánchez Román Instituto de Ciencias Médicas y Nutrición Salvador Zubirán Ciudad de México. Natasha Alcocer Castillejos Instituto de Ciencias Médicas y Nutrición Salvador Zubirán Ciudad de México. Paulina Quiroz Friedman Instituto de Ciencias Médicas y Nutrición Salvador Zubirán Ciudad de México. Cecilia Peñacoba Puente Universidad Rey Juan Carlos Patricia Catalá Mesón Universidad Rey Juan Carlos

\section{resumen/ahstract:}

El cáncer de mama es un tumor maligno y su impacto psicológico ha sido demostrado ampliamente. Tal afección hace evidente la necesidad de trabajar desde la multidisciplinariedad para mejorar la sintomatología afectiva y la limitación funcional de estas pacientes. En el presente trabajo se implementa una intervención psicológica en 69 mujeres con cáncer de mama siendo 19 españolas y 50 mexicanas que fueron divididas en grupo experimental ( $N=41$ ) y control (N=28). El grupo experimental realizó un programa de 4 módulos: 1) manejo de estrés (incorporando ejercicios de consciencia corporal, yoga y mindfulness), 2) aceptación a la enfermedad, 3) regulación emocional, y 4) apoyo social. Los resultados revelaron una mejora de la calidad de vida y de la imagen corporal, disminución de la alexitimia y la sintomatología física y emocional. Estos datos confirman que el programa ofrece resultados beneficiosos para las mujeres con cáncer de mama incorporando el ejercicio físico junto con las técnicas psicológicas.

Breast cancer is a malignant tumor and the psychological impact is demonstrated empirically in the affected people. This condition makes evident the need to work multidisciplinary to help these patients. The present study implements a psychological intervention with 69 women with breast cancer. 19 women are Spanish and 50 women are Mexican. The participating sample was divided into experimental group ( $N=41)$ and control group ( $N=28)$. The experimental group did a 4 module program: 1) stress management (with body awareness exercises, yoga and mindfulness), 2) acceptance of cancer, 3) emotional regulation, and 4) social support. The results revealed an improvement in the quality of life and body image and a decrease in physical and emotional symptoms and alexithymia. Our study confirms that the intervention offers beneficial results for women with breast cancer incorporating physical exercise and psychological techniques. 


\section{palabras clave/keywords:}

Cáncer de mama, intervención psicológica, calidad de vida.

Breast cancer, psychological intervention, quality of life.

\section{Introducción}

El cáncer ginecológico es el más frecuente en la población femenina. En concreto, el diagnóstico del cáncer de mama ha aumentado un 12\% durante el 2018 en países como España o México, lo que lleva a considerar el cáncer de mama como un problema de salud pública relevante (Sociedad Española de Oncología Médica, 2019). Precisamente es en personas afectadas de cáncer de mama donde se han realizado mayores estudios a través de evaluaciones psicológicas que han valorado el impacto que tiene la enfermedad en la calidad de vida percibida relacionada con la imagen corporal y el tratamiento médico recibido (Boing et al., 2018; Teo et al., 2016). Este constructo requiere una gran relevancia al dotarse de un significado subjetivo y personal y que repercute en la salud física, psicológica y social.

En relación con las consecuencias emocionales, son la ansiedad y la depresión los síntomas psicológicos más recurrentes en las mujeres afectadas (Teo et al., 2016) repercutiendo en la disminución de la calidad de vida (Goyal, Levine, Van Zee, Naftalis y Avis, 2018). Estudios previos muestran que la aparición de síntomas depresivos se asocia en mayor medida a la apariencia física tras someterse a una cirugía por extirpación del pecho o de los ganglios afectados. Además, la distorsión de la imagen corporal, el impacto por la pérdida del pelo, la disminución de la funcionalidad del brazo de la axila afectada tras la cirugía o los síntomas físicos como puede ser la fatiga o el dolor durante el tratamiento médico, son factores que aumentan la probabilidad y el mantenimiento de los problemas emocionales (Matthews, Grunfeld y Turner, 2017).

Uno de los factores que se ha tenido en consideración para determinar la intensidad emocional con la que viven estas pacientes todas las experiencias asociadas al cáncer es el ajuste mental que permite adaptarse a esta nueva realidad (Brandao, Schulz y Matos, 2017). Por ajuste mental se entiende la utilización de diferentes estrategias de afrontamiento llevadas a cabo permitiendo una adaptación psicológica ante situaciones que suponen un alto nivel de estrés como pueden ser las revisiones médicas, las sesiones de tratamiento médico, la cirugía, etc. (Yildirim et al., 2018). En varias revisiones sistemáticas sobre el afrontamiento ante el cáncer de mama, coinciden en que el papel de la regulación y expresión emocional facilita la puesta en marcha de estrategias adaptativas que permiten integrar la enfermedad como un hecho superable y que facilite un aprendizaje personal a las mujeres (Brandao, Tavares, Schulz y Matos,2017). Por el contrario, diferentes estudios ponen de manifiesto que la incapacidad de identificar y expresar sentimientos y emociones (alexitimia), es una de las características que definen a esta población y que, a su vez, supone un factor de riesgo en la aparición de trastornos en el estado de ánimo una vez superada la enfermedad (Marrazzo, et al., 2016). 
Considerando el perfil psicológico que presentan estas mujeres, parte de la literatura se ha dedicado a describir diferentes programas de intervención psicológica que abordan las carencias a nivel emocional y físico durante el proceso de enfermedad así como implementar hábitos saludables como la dieta o el ejercicio físico (Xiao et al., 2017). La mayoría de las intervenciones basadas en el ejercicio físico para cáncer de mama, se centra en la implementación de la práctica física en mujeres supervivientes. Sin embargo, un metaanálisis reciente analiza la eficacia de las intervenciones en ejercicio físico con pacientes que están atravesando la enfermedad confirmando sus beneficios en la reducción de los niveles de fatiga provocados por el tratamiento adyuvante (van Vulpen et al., 2016). La confirmación de los resultados positivos de este tipo de intervenciones, impulsan a que la implementación del ejercicio físico en pacientes oncológicos resulte fundamental para la mejora de la funcionalidad física y la calidad de vida (Antunes et al., 2019).

Por otro lado, nuevas técnicas psicológicas centradas en la aceptación y en la atención plena (mindfulness) son cada vez más utilizadas tanto en mujeres que se encuentran en el proceso de enfermedad como aquellas supervivientes (Cifu, Power, Shomstein y Arem, 2018). El mindfulness ha sido el que más apoyo ha recibido por parte de la investigación a través del diseño y la aplicación de programas de intervención y tiene como eje central la psicoeducación, los ejercicios de bodyscan (meditación centrada en la conciencia corporal), la meditación basada en la conciencia plena de la respiración y en la conducta de caminar y en ejercicios de estiramientos y yoga. Los resultados encontrados en los pacientes oncológicos que cumplimentan programas que incorporan el mindfulness son beneficiosos en relación al manejo de las propias emociones así como la mejora de la sintomatología física (Zainal, Booth y Huppert, 2013) puesto que permite abordar la atención a las propias emociones junto a la influencia de la movilidad a nivel físico (estiramientos).

En primer lugar, el presente estudio tiene como objetivo cubrir las carencias que señala la literatura implementando una intervención multicomponente que incluya por un lado técnicas psicológicas y, por otro, ejercicios físicos adaptados con el fin de mejorar la funcionalidad de pacientes diagnosticadas de cáncer de mama en estadios del I al III. En segundo lugar, se pretende obtener una mejora del nivel de calidad de vida percibido así como de la sintomatología física y psicológica.

\section{Método}

\section{Participantes}

Un total de 69 mujeres (50 de nacionalidad mexicana y 19 de nacionalidad española), cumplieron con los siguientes requisitos para entrar como participantes de la investigación:

Ser mayor de edad.

Firmar tanto la hoja de información a la participante como el consentimiento informado.

Ser pacientes referidos de los servicios de oncología que colaboraron en el estudio y que confirmasen el diagnóstico histológico de cáncer de mama invasor en estadios del I al III (no metastásico). 
Encontrarse en tratamiento médico activo (quimioterapia, radioterapia u hormonoterapia).

Ser conscientes de padecer la enfermedad.

Tabla 1.- Datos sociodemográficos y clínicos de las participantes

\section{País}

España (\%)
$\mathrm{n}=19$
$(27.5)$

\section{Estado civil (\%)}

Soltera

Casada

Divorciada

Viuda

Unión libre

$$
\begin{gathered}
\mathrm{n}=16(23.2) \\
\mathrm{n}=36(52.2) \\
\mathrm{n}=9(13.00) \\
\mathrm{n}=4(5.8) \\
\mathrm{n}=4(5.8)
\end{gathered}
$$

Sin estudios

Nivel educativo (\%)

Primaria

$\mathrm{n}=1(1.4)$

$\mathrm{n}=4(5.8)$

Secundaria

$\mathrm{n}=12(17.4)$

Bachillerato

$\mathrm{n}=15(21.7)$

Estudios universitarios

$\mathrm{n}=37(53.5)$

Ocupación laboral (\%)

Profesionales

$\mathrm{n}=16(23.5)$

Educadoras

$\mathrm{n}=3(4.4)$

Comerciantes

$\mathrm{n}=6(8.8)$

Servicios domésticos

$\mathrm{n}=21(30.8)$

No trabaja

$\mathrm{n}=23(33.7)$

Diagnóstico clínico según el estadio de la enfermedad (\%)
IA-IB
$\mathrm{n}=23(34.3)$
IIA-IIB
$\mathrm{n}=29(43.3)$
IIIA-IIIB
$\mathrm{n}=15(22.4)$ 
Del mismo modo, también tenían que cumplir con los siguientes criterios de exclusión:

Ser menores de edad.

Encontrarse con un diagnóstico de cáncer de mama en fase avanzada (enfermedad metastásica o recaída de la enfermedad).

Encontrarse en situaciones difíciles que les impidieran contestar por sí mismas a los instrumentos de la evaluación (como por ejemplo ceguera, delirium, analfabetismo, etc.).

No firmar el consentimiento informado, ni la hoja de información a la participante.

Contar con un diagnóstico psiquiátrico grave como psicosis o trastorno bipolar entre otros.

Las características sociodemográficas y médicas de la muestra, aparecen recogidas en la Tabla 1.

\section{Procedimiento}

El presente estudio contó con la aprobación del Comité de Ética de la Facultad de Ciencias de la Salud de la Universidad Rey Juan Carlos (Alcorcón, Madrid), del Hospital Universitario de Móstoles (Madrid) y del Instituto de Ciencias Médicas y Nutrición Salvador Zubirán (Distrito Federal, México). Los profesionales de cada una de las unidades de oncología facilitaron los datos personales de las pacientes interesadas en el estudio para contactar con ellas telefónicamente y concretar una cita para realizar una evaluación individual en la que mediante una entrevista se les administraba una serie de cuestionarios (evaluación pre). Tras la evaluación, las participantes fueron asignadas de manera aleatoria a la condición control y a la condición experimental. Aquellas mujeres que formaban parte del grupo experimental, acudían un día a la semana a las sesiones de las que constaba el programa terapéutico durante 16 semanas. Pasado este tiempo, tanto el grupo experimental que había realizado el programa como el grupo control realizaban de nuevo la evaluación individual (evaluación post).

\section{Instrumentos}

Tanto el protocolo de evaluación pre como el post, contaban con una serie de preguntas abiertas para recoger información acerca de variables sociodemográficas (edad, país de procedencia, estado civil, laboral y educativo) y de variables médicas referentes al estadio de la enfermedad. Sobre las variables de estudio, cabe destacar que todas se evaluaron por medio de cuestionarios de autoinforme cuyas respuestas se obtienen mediante escalas de tipo Likert. Para facilitar la recogida de información sobre las variables de estudio, se dividieron en cinco bloques: un primer bloque corresponde a la calidad de vida; el segundo bloque a la imagen corporal; el tercer bloque a la afección emocional incluyendo el afecto positivo y negativo, la alexitimia y los síntomas de ansiedad y depresión; un cuarto bloque se centra en la sintomatología física y, por último, el quinto bloque corresponde al afrontamiento ante la enfermedad.

La calidad de vida se evaluó por medio de la validación española del Cuestionario de Calidad de Vida EORTC-QLQ-C30 (Arraras et al., 2008). En la escala que mide el índice gene- 
ral de la calidad de vida, una mayor puntuación indica una mejora de la misma. Este cuestionario ha mostrado tener una buena consistencia interna (Alpha de Cronbach de 0.70).

Para evaluar la percepción que tienen las mujeres afectadas de su propio cuerpo se utilizó la Escala de Imagen Corporal de Hopwood, Fletcher, Lee y Ghazal (2001). En población hispano hablante no existe una validación consolidada de este instrumento, pero en revisiones anteriores realizadas por Sebastián, Manos, Bueno y Mateos (2007), se confirma que este instrumento es válido en este tipo de población teniendo un Alpha de 0.85.

La afección emocional se evaluó por medio de tres cuestionarios. Para medir la sintomatología ansiosa y depresiva se utilizó la versión española de la Escala Hospitalaria de Ansiedad y Depresión de Hamilton (Tejero, Guimerá y Farré, 1986), la cual consta de una subescala de depresión centrada en la anhedonia como síntoma principal que diferencia la depresión de la subescala de ansiedad. Este instrumento muestra una alta consistencia interna con Alphas entre el 0.83 y el 0.85 y altas puntuaciones en ambas escalas indican mayor afección ansiosa y/o depresiva. Por otro lado, se empleó la versión española de la Escala de Afecto Positivo y Afecto Negativo (SPANAS) (Sandín et al., 1999) para explorar el estado de ánimo por medio de emociones y sentimientos tanto positivos como negativos. Mayores puntuaciones en cada subescala indican mayor afecto tanto positivo como negativo. La consistencia interna para cada constructo del instrumento es alta, siendo para el afecto negativo un alpha entre 0.84-0.87 y para el afecto positivo un alpha entre 0.86-0.90. Por último, la alexitimia se midió con la validación española de la Escala de alexitimia de Toronto TAS20 (Paéz et al., 1999) para evaluar la dificultad de conexión con las propias emociones. Mayores puntuaciones indican mayor dificultad emocional. La consistencia interna de la escala general de alexitimia para población femenina en España es de 0.76 mientras que en México es de 0.85 .

Con respecto a la sintomatología física, se evaluó por medio de las escalas referentes a los síntomas del Cuestionario de Calidad de Vida EORTC-QLQ-C30 (fatiga, dolor, náuseas y vómitos, disnea, insomnio, anorexia, estreñimiento, diarrea e impacto económico). Para ampliar la información, se incluyó un módulo complementario del Cuestionario de Calidad de Vida de Cáncer de Mama EORTC-QLQ-BR23 (Arraras et al., 2001) que es exclusivo para evaluar los síntomas específicos en estas pacientes (efectos secundarios del tratamiento médico, afección en la imagen corporal, funcionamiento sexual, disfrute del sexo y perspectiva que tienen las pacientes respecto al futuro). Este cuestionario específico para la calidad de vida en cáncer de mama cuenta con un Alpha de Cronbach de 0.70, en las escalas de sintomatología una mayor puntuación indica una mayor afección.

Por último, las estrategias de afrontamiento hacia el cáncer de mama se evaluaron a través de la validación española de la Escala de Ajuste Mental al Cáncer (MAC) (Ferrero, Barreto y Toledo, 1994). Este instrumento está compuesto de cinco dimensiones que corresponde a las siguientes estrategias cognitivas: espíritu de lucha, desesperanza, preocupación ansiosa, fatalismo y evitación. La consistencia interna mediante el coeficiente de alpha de Cronbach osciló entre 0.65 y 0.84 . Altas puntuaciones en cada una de las escalas indica un mayor uso de dichas estrategias de afrontamiento. 


\section{Programa de intervención}

La intervención psicológica que realizaban las mujeres que formaban parte del grupo experimental consta de 16 sesiones en formato individual y de aproximadamente una hora y media de duración. Las sesiones se dividen en cuatro módulos de trabajo de cuatro sesiones cada uno que siguen el siguiente orden: 1) manejo del estrés, 2) aceptación de la enfermedad, 3) regulación emocional y 4) apoyo social.

A continuación, se describirán cada uno de los módulos de trabajo junto con los objetivos que se pretenden alcanzar en cada uno de ellos.

\section{Módulo de manejo del estrés}

Las sesiones que engloban este módulo se basan en el modelo teórico de la adaptación de Moorey y Greer (1989) que sostiene que la evaluación cognitiva que se realiza del propio diagnóstico se percibe como un reto capaz de activar estrategias de afrontamiento, los cuales pueden tener un resultado emocional determinado. Estas estrategias de afrontamiento que proponen, determinarán cinco tipos o estilos de afrontamiento existentes: espíritu de lucha, evitación o negación, fatalismo o aceptación estoica, desamparo (indefensión) y preocupación ansiosa. Los objetivos que se persiguen en este módulo son los siguientes:

Ofrecer a la mujer una orientación psicoeducativa acerca de lo que es el distrés y el eustrés para que identifique los elementos que desencadenan su respuesta.

Fomentar el autoconocimiento para reconocer su forma de reaccionar ante situaciones estresantes.

Facilitar nuevas estrategias para afrontar las situaciones adversas que pueden rodear a la enfermedad.

Enseñar técnicas de afrontamiento ante el estrés por medio del mindfulness como método para enseñar la diferencia entre mente y cuerpo, guiando a un equilibrio entre ellos que beneficie su bienestar.

Reforzar lo aprendido en cada sesión por medio de tareas para casa.

Dentro de este módulo, una de las cuatro sesiones se centra exclusivamente a la atención corporal mediante ejercicios básicos de yoga y estiramientos que permitan a las mujeres a concienciarse sobre el estado basal de su organismo y su estado emocional. El objetivo de dicha sesión se centra en la mejoría indirecta de la funcionalidad de las extremidades por medio del mindfulness y posturas básicas de yoga.

\section{Módulo de aceptación ante la enfermedad}

En este segundo módulo se pretende trabajar en la autocompasión bajo la filosofía mindfulness, siguiendo el modelo de aceptación de Germer y Simón (2011). Este modelo explica que la aceptación incondicional ante la situación se persigue por medio de varios pasos: el manejo de la resistencia, la apertura con actitud curiosa a las emociones, la tolerancia al dolor emocional, el permiso al flujo del contenido interno y, por último, el permiso de poder abrazar todas las experiencias que surjan. Los objetivos que se pretenden conseguir son los siguientes: 
Facilitar un trabajo psicoeducativo de lo que consiste la aceptación de la situación en el presente, diferenciándolo de la resignación.

Detectar barreras psicológicas impuestas por la mujer y que puedan impedir la orientación de su conducta hacia su propia satisfacción.

Manejo de la autoestima y de la autoeficacia.

Describir los valores personales que orienten la conducta que produzca satisfacción.

Ofrecer un espacio para el diálogo que permita el manejo del propio perdón y de la gratitud hacia sí misma por el trabajo personal realizado.

Fomentar la reflexión personal a través de las tareas mandadas para casa.

\section{Módulo de regulación emocional}

El siguiente módulo permite ahondar en el mundo emocional dotándole del sentido que pueda tener para la mujer las emociones que está experimentando en el momento actual. El trabajo dentro de este módulo se centra en las ideas básicas del modelo teórico de Mayer y Salovey sobre la inteligencia emocional (1997), el cual explica que la habilidad para manejar las emociones y discriminarlas, ayudan a la persona a dirigir sus pensamientos y sus acciones. Los objetivos que se cubren con las cuatro sesiones sobre regulación emocional son los siguientes:

Comprender, desde una perspectiva psicoeducativa, la importancia de las emociones.

Percibir y expresar las emociones de una manera adecuada.

Detectar la dirección del pensamiento en función de la emoción experimentada en ese momento.

Comprender el propio estado emocional por medio de las etiquetas de las diferentes emociones.

\section{Módulo de apoyo social}

Las últimas cuatro sesiones del programa, se dedican a trabajar la importancia que tiene el apoyo social percibido por las mujeres en el manejo de situaciones estresantes relacionadas con la enfermedad, basándose en el modelo teórico del efecto amortiguador del apoyo social de Cohen y McKay (1984). Los objetivos que se alcanzan en este módulo son los siguientes:

Identificar y seleccionar los miembros que conforman la red de apoyo.

Determinar qué tipo de apoyo facilita cada uno de los miembros en situaciones estresantes. Promoción de la comunicación asertiva ante situaciones conflictivas.

\section{Análisis estadísticos}

Para los análisis estadísticos, se utilizó el programa estadístico IBM SPSS Statistics para Windows, Versión 20.0. Armonk, NY: IBM Corp. En primer lugar, se realizaron análisis estadísticos de tipo descriptivo utilizando las variables sociodemográficas y clínicas para la definición de la muestra participante (Tabla 1). En segundo lugar, se procedió a realizar un 
Tabla 2.- Diferencias significativas en los grupos experimental y control

\begin{tabular}{|c|c|c|c|c|c|c|}
\hline \multicolumn{4}{|c|}{$\begin{array}{l}\text { Grupo Experimental } \\
\qquad(n=41)\end{array}$} & \multicolumn{3}{|c|}{$\begin{array}{l}\text { Grupo Control } \\
\qquad(\mathrm{n}=28)\end{array}$} \\
\hline & $\begin{array}{c}\text { Pre } \\
\text { M (DE) }\end{array}$ & $\begin{array}{c}\text { Post } \\
\text { M(DE) }\end{array}$ & $t$ & $\begin{array}{c}\text { Pre } \\
\text { M(DE) }\end{array}$ & $\begin{array}{c}\text { Post } \\
\text { M(DE) }\end{array}$ & $t$ \\
\hline \multicolumn{7}{|c|}{ Índice de calidad de vida } \\
\hline & $62.39(27.43)$ & $\begin{array}{c}74.76 \\
(20.15)\end{array}$ & .002 & $64.23(24.01)$ & $73.35(23.65)$ & .130 \\
\hline \multicolumn{7}{|c|}{ Imagen corporal } \\
\hline & $12.28(8.28)$ & $\begin{array}{c}9.93 \\
(6.39)\end{array}$ & .020 & $13.27(7.56)$ & $12.69(7.01)$ & .632 \\
\hline \multicolumn{7}{|c|}{ Variables emocionales } \\
\hline Afecto Positivo & $34.35(8.26)$ & $\begin{array}{l}37.53 \\
(7.00)\end{array}$ & .006 & $33.88(7.30)$ & $34.56(7.40)$ & .609 \\
\hline Afecto Negativo & $24.62(9.84)$ & $\begin{array}{l}18.41 \\
(6.48)\end{array}$ & .001 & $22.16(7.96)$ & $20.20(8.54)$ & .112 \\
\hline Alexitimia & $56.95(13.56)$ & $\begin{array}{c}49.65 \\
(12.21)\end{array}$ & .000 & $54.77(13.54)$ & $51.35(10.40)$ & .211 \\
\hline Ansiedad & $8.44(4.01)$ & $\begin{array}{c}6.85 \\
(3.71)\end{array}$ & .047 & $7.51(2.74)$ & $6.97(3.85)$ & .478 \\
\hline Depresión & $6.58(4.30)$ & $\begin{array}{l}4.93 \\
(3.78)\end{array}$ & .022 & $5.39(2.85)$ & $5.15(3.36)$ & .727 \\
\hline \multicolumn{7}{|c|}{ Síntomas Físicos } \\
\hline Insomnio & $48.71(36.55)$ & $\begin{array}{c}32.59 \\
(28.09)\end{array}$ & .009 & $45.83(35.18)$ & $32.07(26.89)$ & .038 \\
\hline Pérdida de pelo & $52.82(34.61)$ & $\begin{array}{c}34.51 \\
(22.34)\end{array}$ & .004 & $49.05(30.87)$ & $27.92(26.41)$ & .003 \\
\hline Disfrute del sexo & $42.28(28.24)$ & $\begin{array}{c}88.59 \\
(42.87)\end{array}$ & .000 & $42.76(17.41)$ & $20.62(63.36)$ & .233 \\
\hline \multicolumn{7}{|c|}{ Afrontamiento ante la enfermedad } \\
\hline Espíritu de lucha & $49.48(7.08)$ & $\begin{array}{l}52.84 \\
(3.76)\end{array}$ & .011 & $49.57(6.19)$ & $51.84(6.85)$ & .061 \\
\hline Indefensión & $10.62(4.94)$ & $\begin{array}{c}8.31 \\
(2.51)\end{array}$ & .005 & $10.22(3.62)$ & $9.23(3.79)$ & .156 \\
\hline $\begin{array}{l}\text { Preocupación } \\
\text { ansiosa }\end{array}$ & $25.08(4.88)$ & $\begin{array}{l}22.13 \\
(4.20)\end{array}$ & .001 & $23.00(4.39)$ & $21.24(3.89)$ & .107 \\
\hline
\end{tabular}


análisis de comparación de medias por medio de la prueba $\mathrm{T}$ de student de muestras independientes para comprobar los cambios encontrados en el pre-post para los grupos control y experimental $(\mathrm{p}<.005)$ (Tabla 2$)$.

\section{Resultados}

Los resultados relacionados con los datos sociodemográficos se encuentran en la Tabla 1, no encontrándose diferencias significativas entre las mujeres españolas y mexicanas. En los resultados, se observa que la mayoría son casadas (52.2\%), tienen estudios universitarios (53.5\%), pero no trabajan o bien por encontrarse de baja laboral o estar en situación de desempleo (33.7\%). Referente a las variables médicas, se recogieron datos sobre el estadio de la enfermedad viendo que la mayoría de las mujeres se encontraban en un estadio moderadamente avanzado (estadio IIA-IIB) (43.3\%).

En cuanto a las diferencias encontradas en relación al grupo de pertenencia (control y experimental), en la Tabla 2 pueden observarse las diferencias significativas encontradas en ambos grupos con respecto a los resultados pre y los resultados post con una $\mathrm{p}<.005$.

Como puede verse en la Tabla 2, existe una mejoría entre el pre y el post del grupo experimental en todas las variables de estudio. Respecto al grupo control, únicamente se observa mejoría en el impacto que ejerce la pérdida de pelo por el tratamiento médico y el insomnio.

\section{Discusión}

En este apartado se procederá a debatir los resultados encontrados tomando como punto de partida la literatura previa y el objetivo principal de este trabajo.

En primer lugar, se observa una mejoría en el nivel de calidad de vida percibido en aquel grupo que cumplimenta todas las sesiones del programa de intervención en comparación con el grupo control. Apoyando las conclusiones de investigaciones anteriores, este estudio confirma que el trabajo terapéutico desde una perspectiva multicomponente, mejora la calidad de vida durante la enfermedad perdurando ese beneficio en la fase de supervivencia (Xiao et al., 2017). Estudios previos han encontrado resultados similares referente a los beneficios que aportan las intervenciones psicológicas que incluyen el ejercicio físico adaptado (Witek, Tell y Matthews, 2019). En concreto, aquellos programas de intervención psicoeducativa que trabajan la expresión emocional tienen un beneficio mayor en la calidad de vida de las pacientes durante la etapa de recuperación comprobándose el mantenimiento de dichos cambios un año después de cumplimentar el programa físico y psicológico (Zainal, Booth y Huppert, 2013).

En lo que se refiere a la imagen que las mujeres tienen de sí mismas, se observa un cambio positivo tras la intervención psicológica en la condición experimental, así como una disminución de la afección emocional que manifestaban en las evaluaciones preliminares. Tal y como señalan Boing et al. (2018), los cambios físicos consecuentes de la enfermedad generan una gran perturbación emocional en las pacientes puesto que la apariencia física tras la cirugía y el tratamiento entra en conflicto con el propio concepto que tienen sobre la mujer, lo que también disminuye la percepción de su propia calidad de vida (Teo et al., 2016). El presente estudio también muestra que las mujeres recuperan la funcionalidad en 
sus actividades cotidianas. Este cambio tras el programa también repercute en la percepción que conciben del propio cuerpo y, como consecuencia mejoran en las relaciones mantenidas con su entorno más cercano (módulo de apoyo social).

Resaltando la importancia del manejo emocional en la identificación y expresión de las emociones, se observa una disminución de los niveles de alexitimia en las mujeres del grupo experimental tras el paso por el programa. Este resultado implica que una vez finalizado el programa, las mujeres muestran un mayor conocimiento de su mundo emocional detectando lo que les ocurre para poder plantear recursos adaptativos ante las situaciones a las que se someten a causa de la enfermedad. Existen pocos estudios acerca de la influencia de la alexitimia en el cáncer de mama (Marrazzo, et al., 2016), sin embargo, los resultados confirman el beneficio que reportan las mujeres tras el trabajo terapéutico durante su recuperación. Por otro lado, los resultados confirman a su vez la mejora de los síntomas ansiosos y/o depresivos. Además, las mujeres que participan en el programa, mejoran en la experimentación de emociones de carácter positivo generando sensaciones de placer y satisfacción (afecto positivo). El trabajo psicológico centrado en la identificación y etiquetación emocional junto con los ejercicios de yoga aprendidos en el módulo de manejo de estrés, favorecen el alivio personal por mantener el control del propio estado psicológico así como la mejora de la condición física (Cifu et al., 2018).

En cuanto a la sintomatología física, este estudio muestra que la intervención multicomponente muestra una reducción de los problemas de insomnio, así como el impacto que conlleva la pérdida de pelo tras el tratamiento médico. En comparación a otros síntomas físicos que suelen ser relevantes en el cáncer de mama como puede ser el dolor o la fatiga, nuestro estudio manifiesta la importancia de atender la irrupción del sueño que tienen estas mujeres ya que se suele relacionar con problemas de depresión, reducción de la calidad de vida y aparición de fatiga en la fase de supervivencia (Goyal et al., 2018). La inclusión del ejercicio físico en el programa junto al trabajo psicológico apoya los resultados encontrados en un metaanálisis reciente en donde se confirma que la práctica de ejercicio físico adaptado mejora, en mayor medida, la conciliación del sueño ininterrumpido por las noches (Mercier, Savard y Bernard, 2017). Como novedad añadida a la literatura previa sobre este problema de salud, el estudio ha detectado una mejoría en un aspecto de la sexualidad como es el disfrute de las relaciones sexuales mantenidas con sus parejas. Estos resultados apoyan la necesidad de trabajar este aspecto en las mujeres puesto que suele ser una demanda que no solo se presenta tras la cirugía, sino que persiste durante toda la fase de recuperación hasta la supervivencia y que, además, repercute en la relación de pareja.

Respecto a las estrategias de afrontamiento que utilizan las mujeres para afrontar la enfermedad, se observan diferencias significativas respecto a las estrategias puestas en marcha antes y después de la intervención. Es decir, las mujeres de la condición experimental manifiestan niveles elevados de rumiación (preocupación ansiosa) así como sentimientos de indefensión antes del programa. Sin embargo, después de la intervención consiguen adquirir una visión de superación de la propia enfermedad que se relaciona con estados de ánimo positivos y empoderamiento por la mejora de la funcionalidad física. Estudios realizados sobre variables psicológicas positivas confirman que es posible que las mujeres puedan 
integrar la enfermedad como una experiencia que les beneficie personalmente en cuanto al aprendizaje de nuevas estrategias de afrontamiento ante situaciones estresantes. Igualmente, el trabajo de la movilidad por medio de la gimnasia adaptada, (ejercicios de pilates o de yoga fundamentalmente), las pacientes se sienten capacitadas para retomar las actividades cotidianas de su vida (Cramer et al., 2012).

Este estudio cuenta con una serie de aportaciones que animan a continuar la línea del diseño y aplicación de programas terapéuticos. Siguiendo el trabajo de autores como Zainal et al. (2013) sobre los beneficios que aporta el mindfulness, este diseño se ha basado en el desarrollo de actividades centradas en la atención plena siguiendo también la metodología cognitivo-conductual, puesto que muestra validez empírica en la mejoría de las pacientes con cáncer de mama (Stagl et al., 2015). En una revisión sistemática realizada por Duijts, Faber, Oldenburg, van Beurden y Aaronson (2011) sobre la eficacia de técnicas conductuales, señalan el ejercicio físico como un tratamiento pautado para estas pacientes que beneficia disminuyendo los niveles de fatiga, depresión, ansiedad y, por otro lado, mejora la condición física repercutiendo en una mejora de la imagen corporal. El yoga es un ejercicio físico que ha sido utilizado para facilitar el trabajo físico por medio de la postura junto con la unión del trabajo psicológico a través de la meditación guiada y el control de la respiración y parece que los resultados obtenidos son claros respecto a la mejora de la sintomatología ansiosa y depresiva a corto plazo (Cramer et al., 2012). Es por ello que en el presente programa de intervención se han aportado algunos ejercicios de yoga ajustados a la funcionalidad de las pacientes para reforzar el trabajo emocional desde la consciencia del cuerpo y las sensaciones experimentadas.

Finalmente, este estudio cuenta con algunas limitaciones. En primer lugar, cuenta con un bajo tamaño muestral que impide la generalización de resultados, por lo que podría solventarse ampliando la muestra de ambos grupos. Otro punto a tratar es el mantenimiento de los resultados en el tiempo puesto que se observa que el grupo control mejora en algunos síntomas físicos evaluados (insomnio e impacto por la pérdida de pelo). Y por último, para conocer la eficacia del programa de intervención diseñado y el mantenimiento de los cambios evaluados en el post en ambos grupos, se plantea realizar evaluaciones de seguimiento adicionales a la evaluación post.

\section{REFERENGIAS}

Antunes, P., Esteves, D., Nunes, C., Joaquim, A., Pimentel, F. L. y Fonseca-Moutinho, J. (2019). Health-related quality of life and physical fitness in breast cancer patients: The impact of a supervised physical exercise program in women with no exercise experience. Psychology, Health \& Medicine, 24(9), 1038-1046. doi.org/10.1080/1 3548506.2019.1597978

Arraras, J.I., Martín Tejedor, J.J.I., Vera, R., Pruja, E.L., Marcos, M., Arias, F. y Valerdi, J.J. (2001). El cuestionario de calidad de vida para cáncer de mama de la EORTC OLQ-BR23: estudio psicométrico con una muestra española. Psicología Conductual, 9(1), 81-97.

Arraras Urdaniz, J.I., Villafranca Iturre, E., Arias de la Vega, F., Domínguez Domínguez, M.A., Lainez Milagro, N., Manterola Burgaleta, A. y otros. (2008). The EORTC Quality of Life Questionnaire QLQ-C30 (Version 3.0). Validation study for spanish prostate cancer patients. Archivos españoles de urología, 61(8), 949-957. 
Boing, L., de Berm Fretta, T., Souza Vieira, M., Andreatta Denig, L., Bergmann, A. y de Azevedo Guimaraes, A.C. (2018). Physical activity, fatigue and quality of life during a clinical adjuvant treatment of breast cancer: a comparative study. Motricidade, 14(2-3), 59-70.doi.org/10.6063/motricidade.13476.

Brandao, T., Shulz, M.S. y Matos, P.M. (2017). Psychological adjustment after breast cancer: A systematic review of longitudinal studies. Psycho-Oncology, 26(7), 917-926.doi:10.1002/pon.4230.

Cifu, G., Power, M.C., Shomstein, S. y Arem, H. (2018). Mindfulness-based interventions and cognitive function among breast cancer survivors: a systematic review. BMC Cancer, 18(1), 1163.doi:10.1186/s12885-018-5065-3.

Cohen, S. y McKay, G. (1984). Social support, stress and the buffering hypothesis: A theoretical analysis. En: A. Braum, S.E. Talor, y J.E. Singer (Eds.), Handbook of Psychology and health. Hillsdale (pp.310-357). New Jersey.

Cramer, H., Lange, S., Klose, P., Paul, A. y Dobos, G. (2012). Yoga for breast cancer patients and survivors: a systematic review and meta-analysis. BMC Cancer, 12, 412.doi:10.1186/1471-2407-12-412.

Duijts, S.F., Faber, M.M., Oldenburg, H.S., van Beurden, M. y Aaronson, N.K. (2011). Effectiveness of behavioral techniques and physical exercise on psychosocial functioning and health-related quality of life in breast cancer patients and survivors- a meta-analysis. Psychooncology, 20(2), 115-126.doi:10.1002/pon.1728.

Ferrero, J., Barreto, M.P. y Toledo, M. (1994). Mental adjustment to cancer and quality of life in breast cancer patients: an exploratory study. Psychooncology, 3(3), 223-232.doi:10.1002/pon.2960030309.

Germer, C. y Simón, V. (2011). Compasión y autocompasión. En V. Simón y C. Germer. (coords.), Aprender a practicar mindfulness (pp.1-20). Barcelona: Sello Editorial.

Goyal, N.G., Levine, B.J., Van Zee, K.J., Naftalis, E. y Avis, N.E. (2018). Trajectories of quality of life following breast cancer diagnosis. Breast cancer research and treatment, 169(1). 163-173.doi:10.1007/s10549-018-4677-2.

Marrazzo, G., Sideli, L., Rizzo, R., Marinaro, A.M., Mulè, A., Marrazzo, A., La Cascia, C. y La Barbera, D. (2016). Quality of life, alexithymia, and defence mechanisms in patients affected by breast cancer across different stages of illness. Journal of Psychopathology 22(2), 141-148.doi: 10.1037/t01318-000.

Matthews, H., Grunfeld, E.A. y Turner, A. (2017). The efficacy of interventions to improve psychosocial outcomes following surgical treatment for breast cancer: a systematic review and meta-analysis. Psycho-Oncology, 26(5), 593-607.doi:10.1002/pon.4199.

Mayer, J.D. y Salovey, P. (1997). “What is emotional intelligence?" En P. Salovey y D. Sluyter (Eds.), Emotional development and emotional intelligence: Implications for educators (pp. 3-31). New York: Basic Books.

Mercier, J., Savard, J. y Bernard, P. (2017). Exercise interventions to improve sleep in cancer patients: A systematic review and meta-analysis. Sleep Medicine Reviews, 36, 43-56.doi:10.1016/j.smrv.2016.11.001.

Moorey, S. y Greer, S. (1989). Psychological therapy for patients with cancer: a new aproach. Londres, Reino Unido: Heinemann Medical Books.

Páez, D., Martínez-Sánchez, F., Velasco, C., Mayordomo, S., Fernández, I. y Blanco, A. (1999). Validez psicométrica de la escala de alexitimia de Toronto (TAS-20): Un estudio transcultural. Boletín de Psicología, 63, 55-76.

Paterson, C., Lengacher, C.A., Donovan, K.A., Kip, K.E. y Tofthagen, C.S. (2017). Body image in younger breast cancer survivors: a systematic review, Cancer Nursing, 39(1), 39-58.doi:10.1097/NCC.0000000000000251.

Sandín, B., Chorot, P., Lostao, L., Joiner, T.E., Santed, M.A. y Valiente, R.M. (1999). Escala PANAS de afecto positivo y negativo: validación factorial y convergencia transcultural. Psicothema, 11(1), 37-51.

Sebastián, J., Manos, D., Bueno, M.J., y Mateos, N. (2007). Imagen corporal y autoestima en mujeres con cáncer de mama participantes en un programa de intervención psicosocial. Clínica y Salud, 18(2), 137-161.

Sociedad Española de Oncología Médica (SEOM), Las cifras del cáncer en España 2019. Recuperado de: https://seom. org/dmcancer/wp-content/uploads/2019/Informe-SEOM-cifras-cancer-2019.pdf.

Stagl, J.M., Bouchard, L.C., Lechner, ,S.C., Blomberg, B.B., Gudenkauf, L.M., Jutagir, D.R., Glück, S., Derhagopian, R.P., Carver, C.S. y Antoni, M.H. (2005). Long term psychological benefits of cognitive behavioral stress management for women with breast cancer: 11 year follow up of a randomized controlled trial. Cancer, 121(11), 1873-1881.doi:10.1002/cncr.29076. 
Tejero, A., Guimerá, E.M. y Farré, J.M. (1986). Uso clínico del HAD (Hospital Anxiety and Depression Scale) en población psiquiátrica: un estudio de su sensibilidad, fiabilidad y validez. Revista Departamento Psiquiatría Facultad de Medicina Barna, 13, 233-238.doi:10.1016/j.rpsm.2012.01.003.

Teo, I., Reece, G.P., Christie, I.C., Guindani, M., Markey, M.K., Heinberg, L.J., Crosby, M.A. y Cororve Fingeret, M. (2016). Body image and quality of life of breast cancer patients: influence of timing and stage of breast reconstruction. Psycho-oncology, 25(9), 1106-1112.doi:10.1002/pon.3952.

van Vulpen, J.K., Peeters, P.H.M., Velthuis, M.J., van der Wall, E. y May, A.M. (2016). Efectos del ejercicio físico durante el tratamiento adyuvante del cáncer de mama en las dimensiones físicas y psicosociales de la fatiga relacionada con el cáncer: un metanálisis. Maturitas ,85, 104-111.doi.org/10.1016/j.maturitas.2015.12.007

Witek Janusek, L., Tell, D. y Mathews, H.L. (2019). Mindfulness based stress reduction provides psychological benefit and restores immune function of women newly diagnosed with breast cancer: a randomized trial with active control. Brain, Behavior and Immunity, 80, 358-373.doi:10.1016/j.bbi.2019.04.012.

Xiao, F., Song, X., Chen, Q., Dai, Y., Xu, R., Qiu, C. y Guo, Q. (2017). Effectiveness of psychological interventions on depression in patients after breast cancer surgery: a meta-analysis of randomized controlled trials. Clinical Breast Cancer 17(3), 171-179.doi:10.1016/j.clbc.2016.11.003.

Yildirim, N.K., Özkan, M., Ilgün, A.S., Sarsenov, D., Alço, G., Aktepe, F., ... Özmen, V. (2018). Possible role of stress, coping strategies, and life style in the development of breast cancer. International Journal of Psychiatry in Medicine, 53(3), 207-220.doi:10.1177/0091217417749789.

Zainal, N.Z., Booth,S. y Huppert, F.A. (2013). The efficacy of mindfulness-based stress reduction on mental health of breast cancer patients: a meta-analysis. Psychooncology, 22(7), 1457-1465.doi:10.1002/pon.3171. 